\title{
The Optimization Problems of Transmit and Receive Design for Radar Operating in Littoral Zones
}

\author{
Yi-nan Zhao, Feng-cong Li, and Zhi-quan Zhou \\ Harbin Institute of Technology, Wenhua West Road No. 2, P.O. Box 55, Weihai, Shandong 264209, China \\ Correspondence should be addressed to Feng-cong Li; xialulee@sina.com
}

Received 3 January 2014; Revised 24 March 2014; Accepted 25 March 2014; Published 17 April 2014

Academic Editor: Zhan Shu

Copyright (c) 2014 Yi-nan Zhao et al. This is an open access article distributed under the Creative Commons Attribution License, which permits unrestricted use, distribution, and reproduction in any medium, provided the original work is properly cited.

\begin{abstract}
The complex environments of the littoral zones prevent the radar from operating efficiently. We propose a waveform and filter design approach to help the radar improve the performance in littoral zones. The approach includes a phase-only nonlinear programming method for suppressing correlation sidelobes in specified Doppler and range intervals, and an alternating projection based algorithm for designing receive filters. Several numerical examples are provided to demonstrate the usage and effectiveness of the proposed methods.
\end{abstract}

\section{Introduction}

Nowadays, radar has been used in many different applications. One of the most intricate working environments for maritime radar is the littoral zone [1-4]. Radars which can work in littoral zones are extremely valuable for national security and military applications. However, a large number of natural and artificial objects exist in littoral zones which degrade the radar performance significantly. Moreover, the radar will suffer performance loss thanks to the mixture of sea and land clutter. The behavior of these clutters are different, and hence it is very difficult to formulate them. For a prosperous coastal city, the massive usage of wireless devices is another threat for radar systems. The existence of numerous wireless communication devices, local radio, and civil and military radars makes the bandwidth resources insufficient. Generally speaking, modern radar technologies are not advanced enough for radars to work efficiently in littoral zones.

Today, many researchers have proposed concepts and methods for developing flexible and multifunctional radar. Considering that the environment is tangled and constantly changing in a littoral zone, the radar systems should be flexible enough to confront all of the possible threats. As mentioned earlier, interested littoral zones are always full of inartificial and man-made objects. The targets with large radar cross-section (RCS) such as wind turbines, ocean liners, beacons, and cliffs will make it very difficult to detect a target with small RCS because of the range sidelobe masking [58]. In fact, range sidelobe masking is a problem which has been researched for a long time. One of the solutions is to design probe waveforms with low correlation sidelobes $[9,10]$. However, it is very difficult to synthesize a waveform with ultralow aperiodic autocorrelation sidelobes [11]. Thus some researchers started to develop algorithms to synthesize waveforms with suppressed sidelobes in desired intervals [1217]. As mentioned in [18], secondary databases are usually available for modern radars [19], and the range intervals in which small targets may exist can be determined using these databases [20]; consequently the probe waveform with low sidelobes in specified intervals can help the radar to operate with efficiency. The correlation magnitude in the suppressed interval of these waveforms is much lower compared with traditional waveforms because the degree of freedom of the waveform design problem is increased. However, the algorithms provided in $[13,17,20]$ did not take the Doppler mismatch into consideration.

In this study, we develop several waveforms and filter design methods for suppressing spectral magnitude in desired bands and correlation sidelobes in specified range and Doppler intervals. The paper is organized as follows. The signal model is provided in Section 2. The methods 
are described in Section 3. Several numerical examples are provided in Section 4.

\section{Signal Model and Problem Statement}

2.1. Notations and Concepts. We denote vectors and matrices with boldface lower and upper case letters, respectively. The imaginary unit is denoted by $j$. The transpose and Hermitian transpose of a vector/matrix are represented by $(\cdot)^{T}$ and $(\cdot)^{H}$, respectively, while $(\cdot)^{*}$ denotes complex conjugate. The $n$th element of a column vector is denoted by $(\cdot)_{n}$. The set-valued function $\operatorname{col}(\cdot)$ represents the column space of a given matrix. The Hadamard product of the vectors/matrices is denoted by $\odot$. The function $\operatorname{dist}(\cdot, \cdot)$ denotes the distance between two mathematical objects. By default, the distance between two vectors is defined by Euclidean norm. For a set $S, \partial S$ denotes its boundary, and $1_{S}$ denotes its indicator function, defined as

$$
1_{S}(x):= \begin{cases}1, & x \in S \\ 0, & x \notin S .\end{cases}
$$

The function/operator composition is defined as

$$
(f \circ g)(x):=f(g(x)) .
$$

Based on composition, the iterated function is defined as follows:

$$
(f)^{K}(x):= \begin{cases}\left((f)^{K-1} \circ f\right)(x), & K>0 \\ x, & K=0 .\end{cases}
$$

An important operator used in this paper is $\operatorname{Diag}(\cdot)$ defined as

$$
\operatorname{Diag}(\mathbf{x}):=\mathbf{I} \odot\left(\mathbf{x} \mathbf{1}^{T}\right)
$$

where I and $\mathbf{1}$ denote identity matrix and all-one vector with appropriate size, respectively. Diag can be used to calculate the element-wise exponential of a given vector by composing the matrix exponential function, which is shown as

$$
(\exp \circ \operatorname{Diag})(\mathbf{x}) \cdot \mathbf{1}=\left[\begin{array}{llll}
e^{(\mathbf{x})_{1}} & e^{(\mathbf{x})_{2}} & \cdots & e^{(\mathbf{x})_{N}}
\end{array}\right]^{T} .
$$

Based on (5), we define a set-valued function that represents all the complex vectors with specified envelope, which is shown as

$$
S_{\mathrm{MC}}(\mathbf{v}):=\left\{(\exp \circ \operatorname{Diag})(j \psi) \cdot \mathbf{v}: \psi \in \mathbb{R}^{N}\right\}, \quad \mathbf{v} \in \mathbb{R}^{N} .
$$

For signal synthesis, $S_{\mathrm{MC}}$ is used extensively in algorithms provided in $[20,21]$.

We introduce the lag shifting matrix $\mathbf{U}_{k} \in \mathbb{R}^{N \times N}$ defined as follows, which is very useful for describing correlation and convolution:

$$
\mathbf{U}_{k}:= \begin{cases}\mathbf{I}_{N \times N}, & k=0 \\
\left(\begin{array}{cc}
\mathbf{0} & \mathbf{I}_{(N-k) \times(N-k)} \\
\mathbf{0} & \mathbf{0}
\end{array}\right), & 0<k<N \\
\mathbf{U}_{-k}^{T}, & -N<k<0 \\
\mathbf{0}_{N \times N} & |k| \geq N .\end{cases}
$$

By extending this matrix, we obtain the lag-frequency shifting matrix $\mathbf{U}_{k, \omega} \in \mathbb{C}^{N \times N}$ which can be used to describe signal correlations with Doppler shift, shown as follows:

$$
\mathbf{U}_{k, \omega}:=\mathbf{U}_{k} \cdot(\operatorname{Diag} \circ \mathbf{f})(\omega),
$$

where $\mathbf{f}: \mathbb{R} \rightarrow S_{\mathrm{MC}}(\mathbf{1})$ is a vector-valued function that represents the complex sinusoid, defined as

$$
\mathbf{f}(\omega):=\left[\begin{array}{lllll}
1 & e^{j \omega} & e^{j 2 \omega} & \cdots & e^{j(N-1) \omega}
\end{array}\right]^{T} .
$$

Based on (7), the cross-correlation of two complex vectors is defined as

$$
c_{n}\left(\mathbf{s}_{1}, \mathbf{s}_{2}\right):=\mathbf{s}_{1}^{H} \mathbf{U}_{n} \mathbf{s}_{2},
$$

and the discrete ambiguity function of a probe waveform is defined as

$$
a_{n, \omega}(\mathbf{s}):=c_{n}(\mathbf{s}, \mathbf{f}(\omega) \odot \mathbf{s})=\mathbf{s}^{H} \mathbf{U}_{n, \omega} \mathbf{s} .
$$

For many topics in signal processing, the optimization problem is usually related to the minimization of errors, such as parameter estimation, filter design, and signal synthesis. A powerful tool for solving this kind of problem is projection onto sets. The projection operation is defined as follows:

$$
\operatorname{proj}(x, S):=\underset{s \in S}{\arg \min } \operatorname{dist}(x, s),
$$

where $\operatorname{proj}(x, S)$ means project element $x$ onto set $S$. The projector is a tool for solving least squares problems if function $\operatorname{dist}(\cdot, \cdot)$ is defined by Euclidean distance.

2.2. The Features of Littoral Zones. Based on the discussion in Section 1, the unique features of the littoral zones can be summarized as follows:

(1) the civil and military wireless devices have significant influence on radar performance;

(2) the radar usually has to detect targets with low RCS from close range; thus, the echo from sea surface, that is, the sea clutter, can degrade the radar performance significantly [22];

(3) numerals natural and artificial objects such as cliffs, reefs, wind turbines, and lighthouses make it difficult to find the interested targets.

These features make the littoral zone a harsh environment for traditional radar. Moreover, the radar scenes are usually quite different when the antenna is aiming in different directions. For example, the direction facing the land and the direction facing the open seas need totally different signal processing methods. Fortunately, the adaptive transmit technologies can be a cure for intricate environments as mentioned in Section 1. In this section, we formulate the problems listed above. By modeling the environment, we can transform the waveform and filter synthesis problem into a mathematical optimization problem. 
2.3. The Mathematical Form of the Probe Waveform and the Receive Filter. The baseband discrete form of a radar waveform can be expressed as a complex vector $\mathbf{s} \in \mathbb{C}^{N}$. Nowadays, many radar systems prefer unimodular waveforms because they can withstand the nonlinear property of the transmitter amplifier and maximize the transmitter efficiency. Thus unimodular is usually a must-have property of radar waveforms, and the synthesis of the probe waveform is equivalent to drawing a vector from $S_{\mathrm{MC}}(\mathbf{1})$ with some good properties. To make our method more adaptable, here we still use $S_{\mathrm{MC}}(\mathbf{v}), \mathbf{v} \in \mathbb{R}^{N}$ to define the waveform, because one may want to synthesize waveforms with specified time domain envelope rather than unimodular waveform in some particular scenarios as follows:

$$
\mathbf{s}=(\exp \circ \operatorname{Diag})(j \psi) \cdot \mathbf{v}, \quad \psi, \mathbf{v} \in \mathbb{R}^{N} .
$$

The synthesis of the probe waveform is also the synthesis of the matched filter. First introduced several decades ago [23], the matched filter has become a standard piece of equipment of many radar systems for it can maintain the total energy of the transmit pulse without degradation of range resolution. Many signal processing procedures utilize the output from the matched filter as their input, and thus the signal model is not only determined by the features of target and interference, but also influenced by the ambiguity function of the probe waveform.

The output from of the matched filter of the $p$ th range cell can be expressed as

$$
r_{\mathrm{MF}}(p)=\sum_{\Delta p=-N+1}^{N-1} \sigma(p+\Delta p) c_{-\Delta p}(\mathbf{s}, \mathbf{f}(\omega(p+\Delta p)) \odot \mathbf{s})
$$

where $\sigma(p+\Delta p) \in \mathbb{R}_{+}$and $\omega(p+\Delta p) \in[-\pi, \pi]$ denote the RCS and Doppler frequency of the object in the $(p+\Delta p)$ th range cell. If a mismatched filter $\mathbf{h}$ is used, the output of the filter is given by

$$
r_{\mathrm{MMF}}(p)=\sum_{\Delta p=-N+1}^{N-1} \sigma(p+\Delta p) c_{-\Delta p}(\mathbf{h}, \mathbf{f}(\omega(p+\Delta p)) \odot \mathbf{s}) .
$$

From (14) and (15), we can find that the correlation sidelobes smear the $p$ th range cell with the energy from the adjacent range cells. The output from the filter can be seen as the convolution of the radar scene and the correlation of the waveform and the receive filter. The principle of the range sidelobe masking phenomenon is shown in Figure 1. To make the problem clear, we do not add noise in this figure.

From Figure 1(a) we can find that if an object with large RCS exists in current scene, it is very difficult to detect small objects around it, and this scenario is very common in littoral zones, for example, a small wooden fishing boat near an ocean liner. For a radar system which can design waveforms on-thefly, this problem can be solved by designing a waveform with correlation nulls, as shown in Figure 1(b). From this figure, we can find that the sidelobe masking is mitigated by using a specifically designed waveform.
2.4. Modeling of Sidelobe Masking. From the discussion above, we can find that if there are strong scatterers near the cell under test (CUT), the sidelobe interference will degrade the performance of the detector. To simplify the notation, here we define the descriptor of strong scatterers. A descriptor is an ordered sequence which is used to describe the vital properties of an interested object. The scatterer descriptor $(\mathrm{SD})$ is defined as

$$
\mathrm{SD}:=(\sigma, p, \omega)
$$

where $\sigma$ represents its RCS, $p$ is the location (range cell index), and $\omega$ is the Doppler frequency. For a given scene, the set $Z_{r}$ denotes all of the strong scatterers' SD. Based on (15), the range sidelobe interference (RSI) in the CUT can be expressed as

$$
\mathrm{RSI}:=\sum_{(\sigma, p, \omega) \in \mathrm{Q}_{r}(i)} \sigma^{2}\left|\mathbf{h}^{H} \mathbf{U}_{p, \omega} \mathbf{s}\right|^{2}
$$

where $i$ is the index of the CUT, $\mathbf{h}$ is the receive filter; for matched filter, $\mathbf{h}=\mathbf{s}$, and $Q_{r}$ is a set-valued function defined as

$$
Q_{r}(i):=\left\{(\sigma, i-p, \omega):(\sigma, p, \omega) \in Z_{r}\right\},
$$

and it is in fact a database of strong scatterers in the current scene. There are many ways to establish this database, for example, utilizing the digital geography map, or other types of sensors. From (17), we can find that for a radar system, the RSI can be mitigated by solving $\min _{\mathbf{s}, \mathbf{h}}$ RSI with some constraints on $\mathbf{s}$ and $\mathbf{h}$.

2.5. Modeling of Active Interference. The bandwidth resources in the littoral zones are insufficient due to the existence of a large number of wireless devices. A traditional radar system will suffer performance loss inevitably. Nowadays, the concepts such as "cognitive" and "adaptive waveform design" have been accepted by the radar community, and many researchers have shown that by designing waveforms with specified spectral shapes, the active interference from other devices can be weakened.

To analyze the spectrum of the signal and interference, we introduce the unit discrete Fourier transform (DFT) matrix. The matrix on $\mathbb{C}^{N}$ is shown as

$$
\mathbf{F}_{N \times N}:=\frac{1}{\sqrt{N}}\left[\mathbf{f}(0) \mathbf{f}\left(\frac{2 \pi}{N}\right) \cdots \mathbf{f}\left(\frac{2 \pi(N-1)}{N}\right)\right]^{H} .
$$

We use the symbol $Z_{f}$ to denote the set comprised of the frequency point (in normalized angular frequency) possessed by other wireless devices. To simplify the notation, we define a new set $Q_{f} \subset \mathbb{N}_{0}$ shown as

$$
Q_{f}:=\left\{\left\lfloor N \frac{\omega}{2 \pi}\right\rfloor: \omega \in Z_{f}\right\} .
$$

The subspace possessed by the interference can be expressed by the following set-valued function:

$$
S_{\mathrm{AI}}\left(Q_{f}\right):=\operatorname{col}\left(\mathbf{F}^{H} \operatorname{Diag}\left(\mathbf{m}\left(Q_{f}\right)\right)\right),
$$




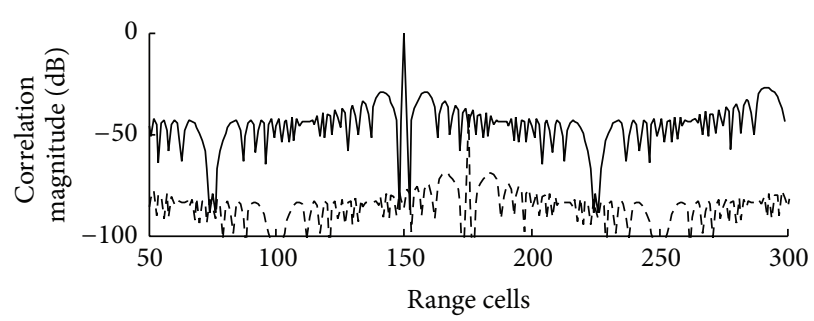

(a)

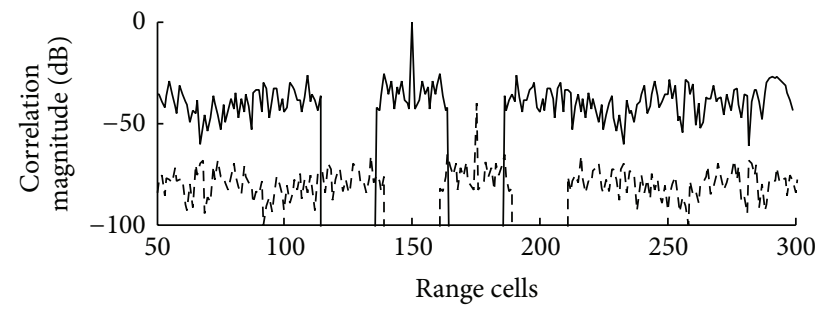

(b)

FIGURE 1: The output from the matched filter without noise. The solid line corresponds to a large target, and the dash line corresponds to a small target. (a) Linear frequency modulation waveform (LFM) is used as the probe waveform and (b) a specifically designed sequence as the proved waveform.

where $\mathbf{m}$ is a vector-valued function defined as

$$
\mathbf{m}(S):=\left[\begin{array}{llll}
1_{S}(0) & 1_{S}(1) & \cdots & 1_{S}(N-1)
\end{array}\right]^{T} .
$$

The mathematical optimization problem for synthesizing a waveform that can suppress active interference is shown as follows [24-26]:

$$
\min _{\mathbf{s}}\left\|\operatorname{proj}\left(\mathbf{s}, S_{\mathrm{AI}}\left(Q_{f}\right)\right)\right\|^{2}
$$

where

$$
\operatorname{proj}\left(\mathbf{s}, S_{\mathrm{AI}}\left(Q_{f}\right)\right):=\mathbf{F}^{H} \operatorname{Diag}\left(\mathbf{m}\left(Q_{f}\right)\right) \mathbf{F} \text {. }
$$

2.6. Modeling of Clutter. The radar systems working in littoral zones usually have to detect objects from close range; consequently, the clutter return becomes the dominant interference. Literature $[27,28]$ has shown that for high resolution radar, the low grazing angle sea clutter becomes spiky, which makes the Gaussian clutter model no longer effective to use. Here we introduce the compound-K model which is a member of the compound-Gaussian family and has been widely used for sea clutter modeling [29, 30].

The compound-Gaussian model assumes that the clutter vector has the following form:

$$
\mathbf{c}=\sqrt{\tau} \mathbf{x},
$$

where $\mathbf{x} \sim \mathscr{C} \mathscr{N}(\mathbf{0}, \mathbf{M})$, and for the compound-K model, the $\operatorname{PDF}$ of $\tau$ is defined as

$$
p(\tau ; b, \nu):=\frac{1}{\left(4 b^{2}\right)^{\nu} \Gamma(\nu)} \tau^{\nu-1} \exp \left(-\frac{\tau}{4 b^{2}}\right) 1_{[0, \infty)}(\tau)
$$

The average clutter to noise ratio (CNR) is defined as follows:

$$
\mathrm{CNR}:=\frac{E\{\tau\}}{\sigma_{n}^{2}}=\frac{4 \nu b^{2}}{\sigma_{n}^{2}} .
$$

The clutter will become spikier if $v$ decreases.

In [17], the clutter spikes are treated as objects with large RCS, and thus the modeling of range sidelobe masking in Section 2.4 is also applicable for modeling spike sidelobe interference. This fact demonstrates that we can use a single method to suppress the range sidelobe interference from large targets and from sea spikes simultaneously.

\section{Waveform and Filter Synthesis}

In Section 2, we formulated the problems for waveform and filter synthesis, and they can be expressed as mathematical optimization problems. For an ordinary optimization problem, the basic components are objective functions and constraint functions. In this section, we derive these functions for waveform optimization and provide the methods for solving the proposed optimization problem.

3.1. Waveform Design for Suppressing Narrow Band Active Interference and Sidelobe Interference. We name the subspace defined by (21) the interference space. To synthesize a waveform with interference suppression capability, we need to solve the following optimization problem:

$$
\begin{array}{ll}
\min _{\mathbf{s}} & J_{f}(\mathbf{s}) \\
\text { s.t. } & \mathbf{s} \in S_{\mathrm{MC}}(\mathbf{v}),
\end{array}
$$

where

$$
J_{f}(\mathbf{s}):=\left\|\operatorname{proj}\left(\mathbf{s}, S_{\mathrm{AI}}\left(Q_{f}\right)\right)\right\|^{2} .
$$

This is a typical constraint optimization problem and can be solved by any constraint optimization algorithm. However, by utilizing the phase-only nonlinear programming (PONLP) technique which is a special case of optimization on the Riemann manifold [31], the constraint problem can be transformed into the following unconstraint optimization problem:

$$
\min _{\psi} J_{f}((\exp \circ \operatorname{Diag})(j \psi) \cdot \mathbf{v})
$$

and the searching procedure of derivative-based algorithms can be expressed as

$$
\mathbf{s}_{k+1}=(\exp \circ \operatorname{Diag})(j \mathbf{d}) \cdot \mathbf{s}_{k}
$$

where $\mathbf{d}$ is the searching vector whose direction is determined by the phase-only derivatives of the objective function.

In addition to the elimination of the modular constraint, another advantage of PONLP is that it transforms the complex vector optimization problem into a real vector optimization problem, and many optimization software packages only 


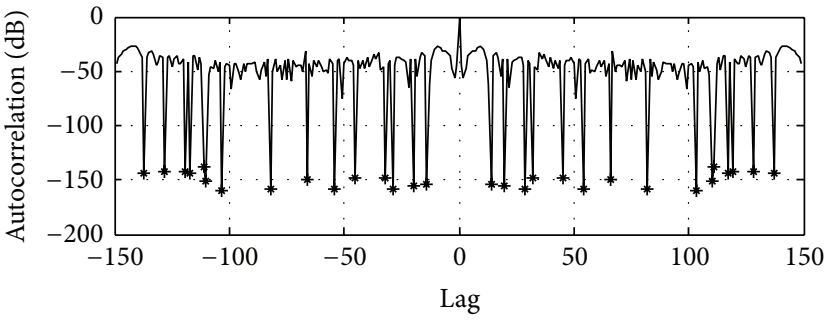

(a)

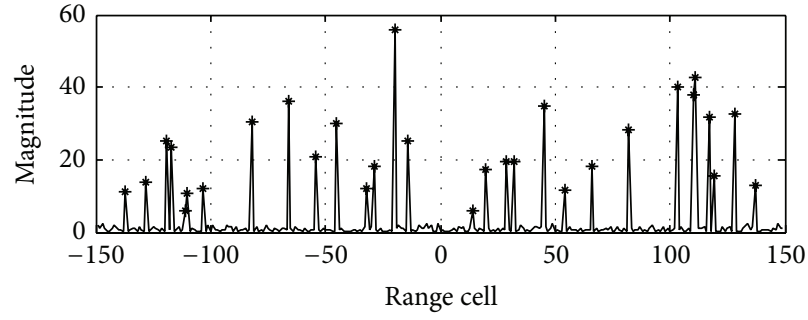

(b)

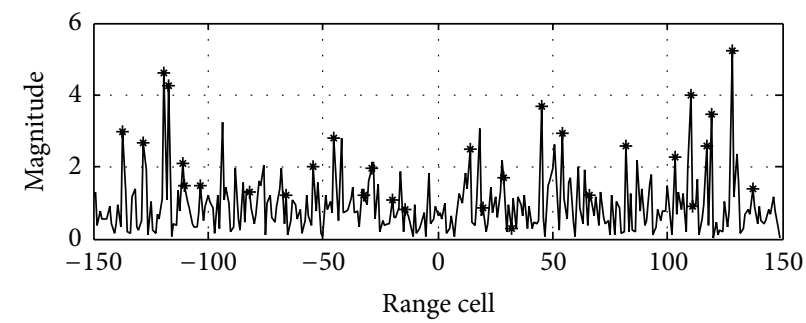

(c)

FIGURE 2: (a) Autocorrelation of a designed unimodular waveform. (b) A range profile of a highly spiky clutter. (c) A range profile of a pervasive clutter.

support the optimization of real vectors. Utilizing the chain rule, the phase-only gradient of (29) is given by

$$
\begin{aligned}
\frac{\partial J_{f}}{\partial \psi} & =\frac{\partial \mathbf{s}^{T}}{\partial \psi} \cdot \frac{\partial J_{f}}{\partial \mathbf{s}}+\frac{\partial \mathbf{s}^{H}}{\partial \psi} \cdot \frac{\partial J_{f}}{\partial \mathbf{s}^{*}} \\
& =2 \operatorname{Im}\left(j \operatorname{Diag}(\mathbf{s}) \operatorname{proj}^{*}\left(\mathbf{s}, S_{\mathrm{AI}}\left(Q_{f}\right)\right)\right)
\end{aligned}
$$

In littoral zones, solving (28) is not enough for synthesizing a practical usable waveform because the correlation sidelobes are not optimized, and the range sidelobe masking will be serious. According to (17), the objective function for suppressing range sidelobe interference is defined as follows if the matched filter is used:

$$
\begin{aligned}
J_{r}(\mathbf{s}) & :=\sum_{(\sigma, k, \omega) \in \mathrm{Q}_{r}(i)}\left|c_{k}(\mathbf{s}, \mathbf{f}(\omega) \odot \mathbf{s})\right|^{2} \\
& =\sum_{(\sigma, k, \omega) \in \mathrm{Q}_{r}(i)} a_{k, \omega}^{*}(\mathbf{s}) a_{k, \omega}(\mathbf{s}) .
\end{aligned}
$$

If the probe waveform must have a specified envelope (usually constant modulus), the waveform synthesis can be described by the following PONLP problem:

$$
\min _{\psi} J_{r}((\exp \circ \operatorname{Diag})(j \psi) \cdot \mathbf{v})
$$

The explicit form of the phase-only gradient is shown as

$$
\begin{aligned}
\nabla_{\psi} J_{r} & =\frac{\partial J_{r}((\exp \circ \operatorname{Diag})(j \psi) \cdot \mathbf{v})}{\partial \psi} \\
& =\sum_{(\sigma, k, \omega) \in \mathrm{Q}_{r}(i)} 2 \operatorname{Re}\left(a_{k, \omega}^{*}(\mathbf{s}) \cdot \nabla_{\psi} a_{k, \omega}(\mathbf{s})\right)
\end{aligned}
$$

where

$$
\begin{aligned}
\nabla_{\psi} a_{k, \omega} & (\mathbf{s}) \\
= & j(\exp \circ \operatorname{Diag})(j \psi) \operatorname{Diag}(\mathbf{v}) \mathbf{U}_{k, \omega}^{H}(\exp \circ \operatorname{Diag})(-j \psi) \mathbf{v} \\
& -j(\exp \circ \operatorname{Diag})(-j \psi) \operatorname{Diag}(\mathbf{v}) \mathbf{U}_{k, \omega}(\exp \circ \operatorname{Diag})(j \psi) \mathbf{v}
\end{aligned}
$$

Notably, for all $r \in \mathbb{R}$, we have

$$
J_{r}((\exp \circ \operatorname{Diag})(j \psi+j r \mathbf{1}) \cdot \mathbf{v})=J_{r}((\exp \circ \operatorname{Diag})(j \psi) \cdot \mathbf{v}),
$$

which means that $r \mathbf{1}$ corresponds to a ridge of the objective function's hyper surface. This is a common feature of many phase-only optimization problems [20].

To yield a waveform that can suppress range sidelobes and active interference simultaneously, we combine $J_{r}$ and $J_{f}$ to form a compound objective function shown as

$$
J_{c}:=\lambda J_{r}+(1-\lambda) J_{f}, \quad \lambda \in[0,1] .
$$

Because the differential operator is linear, the gradient of $J_{c}$ is also the linear combination of the gradients of $J_{r}$ and $J_{f}$.

3.2. The Method for Suppressing Sea Spikes. As mentioned in Section 2.6, the sidelobes of the sea clutter can also be suppressed by solving (30). However, this method is effective only for highly spiky clutter, and Figure 2 demonstrates this fact.

In Figure 2, index 0 corresponds to the CUT, and the asterisks indicate the position of the nulls. In Figure 2(a), the autocorrelation of a designed waveform is shown, and in Figure 2(b), a simulated spiky clutter is given. We can find that only a small number of range cells contain significant 


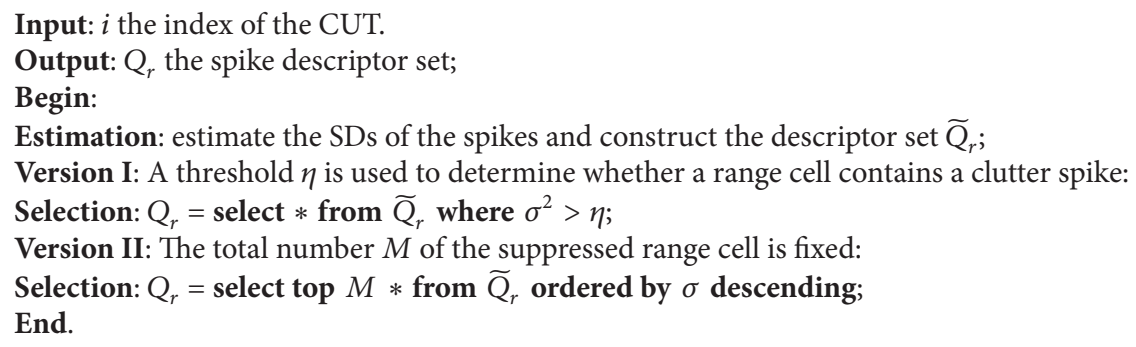

Algorithm 1: Clutter spikes selection algorithm (CSSA).

clutter power. According to (17), for normal waveforms, the sidelobes will smear the energy of spikes on the CUT. However, for the designed waveform shown in Figure 2(a), RSI will be suppressed by the nulls of the waveform autocorrelation. A pervasive clutter is shown in Figure 2(c). Unlike its spiky counterpart, the power of the clutter is distributed homogeneously. Consequently, a small number of autocorrelation nulls cannot remove the main part of the sidelobe interference. It is also obvious that when the number of nulls increases, the average null depth decreases, and thus for a given scenario, if the number of the nulls is too large, the average null depth is not deep enough and the performance is not satisfying. On the other hand, if the nulls are deep enough but too few, there are spikes that still survive and continue to spread their power by range sidelobes on the CUT. In total, the performance of the waveform is related to the clutter property and the number of the nulls.

In order to construct the descriptor set $Q_{r}$, the range cells which contain spikes should be picked up. According to $[32,33]$, the spikes can be identified using three parameters: amplitude, minimum width, and minimum interval. For our approach, considering that the main object is to reduce RSI, we should select range cells with large clutter power. We provide two selection schemes described in SQL style as in Algorithm 1.

After the set $Q_{r}$ is established, we can construct the objective function $J_{r}$ defined in (33) and minimize it to obtain a probe waveform.

Unlike version II of CSSA, the number of the suppressed range cells is determined by multiple factors, including the threshold $\eta$ and the clutter model parameters. Thus we analyze the statistical property of version I based on compound-K model introduced in Section 2.6. According to the selection step of CSSA version I, the probability of a range cell being selected for suppression can be expressed as

$$
P_{S}(\eta)=\int_{\eta}^{\infty} p(\tau ; b, v) d \tau=1-\Gamma_{\text {inc }}\left(\frac{\eta}{4 b^{2}}, v\right)
$$

where $\Gamma_{\text {inc }}$ is the incomplete $\Gamma$ function defined as

$$
\Gamma_{\text {inc }}(a, x):=(\Gamma(a))^{-1} \int_{0}^{x} \exp (-t) t^{a-1} d t .
$$

For a fixed CNR, the shape of $P_{\mathrm{S}}(\eta)$ is affected by $\nu$. To describe the spikiness of the clutter, we define the clutter

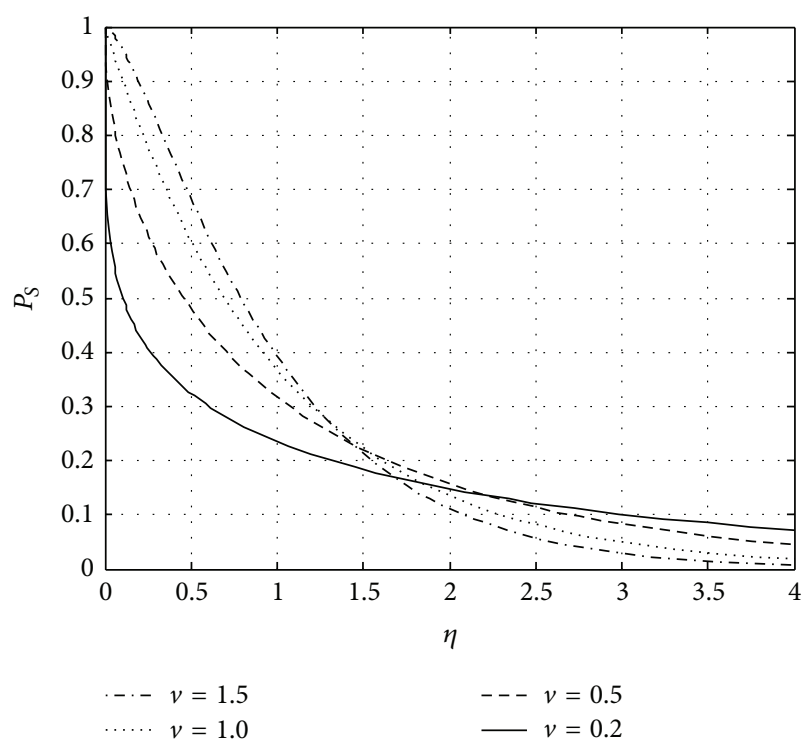

FIgURE 3: $P_{S}$ with respect to $\eta$.

power in the selected range cells to the total clutter power ratio (STR), shown as follows:

$$
\operatorname{STR}(\eta):=\frac{\int_{\eta}^{\infty} \tau p(\tau ; b, v) d \tau}{4 \nu b^{2}}=1-\Gamma_{\mathrm{inc}}\left(\frac{\eta}{4 b^{2}}, \nu+1\right) .
$$

The curves of (39) and (41) are shown in Figures 3 and 4, respectively.

From Figures 3 and 4, we can analyze the performance of the waveform with respect to different clutter parameters. For example, if we set $\eta=2$, and the clutter parameter is $v=0.2$, from Figure 3 we can find that about $15 \%$ range cells should be suppressed, and from Figure 4, we can find that these $15 \%$ range cells comprise over $75 \%$ of the total clutter power. Consequently, the waveform will be very efficient because only a small number of nulls can cancel the main part of the clutter power. However, for $v=1.5$, if we want to suppress $75 \%$ of clutter power, from Figure 3, we can find that nearly $50 \%$ of range cells should be selected. Thus this clutter suppression technique is suitable for highly spiky clutter.

3.3. Filter Design for Suppressing Sidelobes in Desired Doppler Cell. According to (17), the range sidelobes can also be 


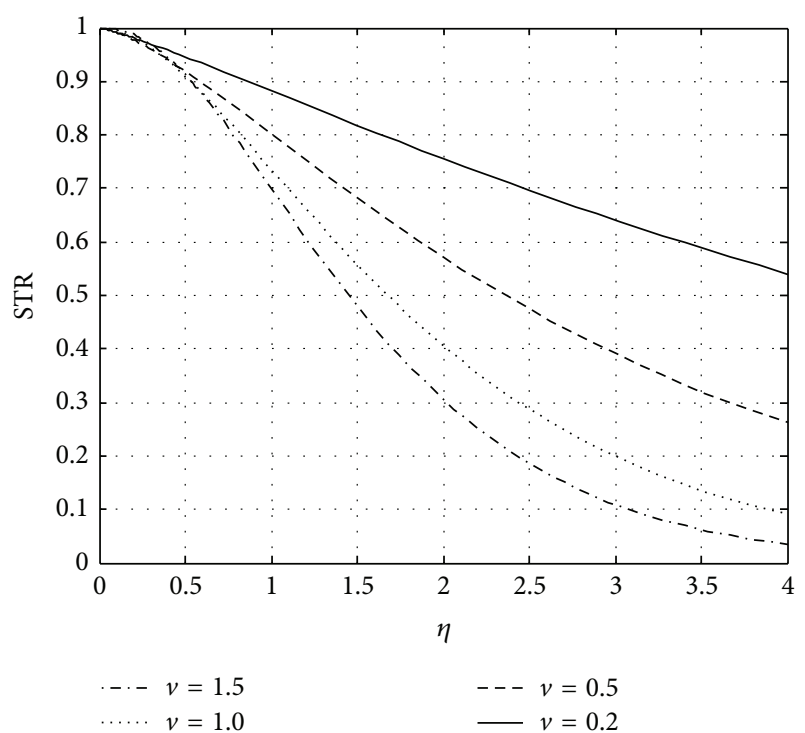

FIGURE 4: STR with respect to $\eta$.

suppressed by filter design. Compared with waveform design, the biggest advantage of filter design is its flexibility. For example, we can pass the receive signal through several different filters simultaneously to suppress different sidelobe zones. The disadvantage is that the SNR loss is inevitable because the Cauchy-Schwarz inequality demonstrates that only the matched filter is optimal under the maximum SNR criterion. Here we develop an algorithm based on the Iterative Spectral Approximation Algorithm (ISAA) framework for filter design [20]. ISAA is a modularized algorithm framework; that is, the framework is comprised of several replaceable modules. By inserting or replacing modules, the algorithm can accomplish different tasks. The reason we choose ISAA is that it is memory efficient. While we have to design multiple filters simultaneously, the memory consumption will become a major concern.

ISAA is based on alternating projections (AP), and thus the basic modules are the sets. For ISAA, there are two kinds of sets: objective sets and constraint sets. The objective sets are comprised of all the vectors with some good property, and the constraint sets are comprised of all the vectors with the musthave property. To derive the objective set for filter design, we derive the objective function first. According to (15), the objective function of the filter design problem is defined as

$$
J(\mathbf{h}):=\left\|\operatorname{Diag}\left(\mathbf{m}\left(Q_{p}\right)\right) \mathbf{c}(\mathbf{h}, \mathbf{f}(\omega) \odot \mathbf{s})\right\|^{2}
$$

where $\omega$ is the normalized angular Doppler frequency of the specified Doppler cell, $\mathbf{m}$ is the vector-valued function defined in (22), and $\mathbf{c}(\mathbf{s}, \mathbf{h})$ is a vector-valued defined as

$$
\begin{aligned}
& \mathbf{c}(\mathbf{s}, \mathbf{h}) \\
& :=\left[\begin{array}{lllllll}
c_{0}(\mathbf{h}, \mathbf{s}) & \cdots & c_{N-1}(\mathbf{h}, \mathbf{s}) & 0 & c_{-N+1}(\mathbf{h}, \mathbf{s}) & \cdots & c_{-1}(\mathbf{h}, \mathbf{s})
\end{array}\right]^{T},
\end{aligned}
$$

and $Q_{p}$ is defined as follows:

$$
Q_{p}:=\left\{(2 N+p) \bmod 2 N:(\sigma, p, \omega) \in Q_{r}(i)\right\} .
$$

Based on the fact shown as follows:

$$
\begin{aligned}
\| \mathbf{m} & \left(Q_{p}\right) \odot \mathbf{c}(\mathbf{h}, \mathbf{f}(\omega) \odot \mathbf{s}) \| \\
= & \left\|\mathbf{m}\left(Q_{p}\right) \odot \mathbf{F}^{H} \operatorname{Diag}^{*}\left(\mathbf{F C}^{T} \mathbf{h}\right) \mathbf{F} \mathbf{C}^{T}(\mathbf{f}(\omega) \odot \mathbf{s})\right\| \\
& =\left\|\widetilde{\mathbf{m}}\left(Q_{p}\right) \odot \mathbf{F}^{H} \operatorname{Diag}^{*}\left(\mathbf{F C}^{T}(\mathbf{f}(\omega) \odot \mathbf{s})\right) \mathbf{F} \mathbf{C}^{T} \mathbf{h}\right\|,
\end{aligned}
$$

where $\widetilde{\mathbf{m}}$ is a vector-valued function shown as

$$
\widetilde{\mathbf{m}}(S):=\left(\begin{array}{cccc}
1 & 0 & \cdots & 0 \\
0 & 0 & \cdots & 1 \\
\vdots & \vdots & . & \vdots \\
0 & 1 & \cdots & 0
\end{array}\right) \mathbf{m}(S),
$$

and $\mathbf{C}$ is the cutoff matrix as follows:

$$
\mathbf{C}:=\left[\begin{array}{ll}
\mathbf{I}_{N \times N} & \mathbf{0}_{N \times N}
\end{array}\right] .
$$

The objective function can be rewritten in the following form:

$$
J(\mathbf{h})=\left\|\operatorname{Diag}\left(\widetilde{\mathbf{m}}\left(Q_{p}\right)\right) \mathbf{F}^{H} \operatorname{Diag}^{*}\left(\mathbf{F C}^{T}(\mathbf{f}(\omega) \odot \mathbf{s})\right) \mathbf{F} C^{T} \mathbf{h}\right\|^{2} .
$$

The solution set of $J(\mathbf{h})=\inf J(\mathbf{h})$, that is, the objective setvalued function, is shown as follows:

$$
\begin{aligned}
& S_{\mathrm{CO}}\left(Q_{p}\right) \\
& \quad:=\operatorname{null}\left(\operatorname{Diag}\left(\widetilde{\mathbf{m}}\left(Q_{p}\right)\right) \mathbf{F}^{H} \operatorname{Diag}^{*}\left(\mathbf{F C}^{T}(\mathbf{f}(\omega) \odot \mathbf{s})\right) \mathbf{F C}^{T}\right) .
\end{aligned}
$$

Its projector is given by

$$
\begin{aligned}
& \operatorname{proj}\left(\cdot, S_{\mathrm{CO}}\left(Q_{p}\right)\right) \\
&:= \mathbf{C F}^{H}\left(\operatorname{Diag}^{*}\left(\mathbf{F C}^{T}(\mathbf{f}(\omega) \odot \mathbf{s})\right)\right)^{-1} \\
& \cdot \mathbf{F}\left(\mathbf{I}-\operatorname{Diag}\left(\widetilde{\mathbf{m}}\left(Q_{p}\right)\right)\right) \\
& \times \mathbf{F}^{H} \operatorname{Diag}^{*}\left(\mathbf{F C}^{T}(\mathbf{f}(\omega) \odot \mathbf{s})\right) \mathbf{F C}^{T} .
\end{aligned}
$$

This projector seems complicated; however, it can be implemented efficiently by fast Fourier transform and Hadamard product for practical usage.

To constraint the SNR loss, we introduce the Inner Product Constraint (IPC) of which the constraint set can be expressed by the following set-valued function:

$$
S_{\text {IPC }}(\mathbf{s}, \xi):=\left\{\mathbf{y} \in \partial S_{\text {ball }}: \frac{|\langle\mathbf{y}, \mathbf{s}\rangle|}{\|\mathbf{s}\|} \geq \xi\right\},
$$

where $S_{\text {ball }}=\left\{\mathbf{x} \in \mathbb{C}^{N}:\|\mathbf{x}\| \leq 1\right\}$. According to (12), the following optimization problem should be solved to obtain the projector onto $S_{\text {IPC }}$ :

$$
\begin{array}{ll}
\min _{\mathbf{y}} & \operatorname{dist}(\mathbf{h}, \mathbf{y}) \\
\text { s.t. } & \mathbf{y} \in S_{\operatorname{IPC}}(\mathbf{s}, \xi) .
\end{array}
$$




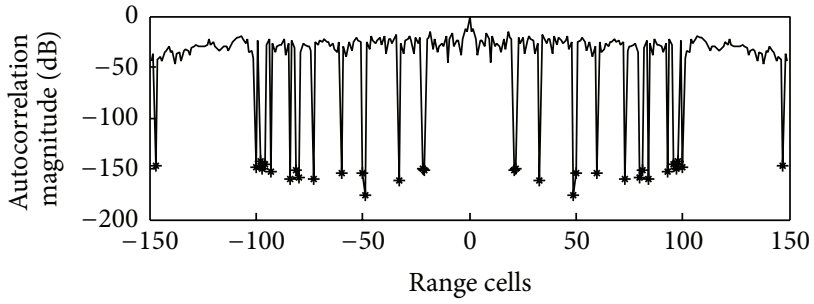

(a)

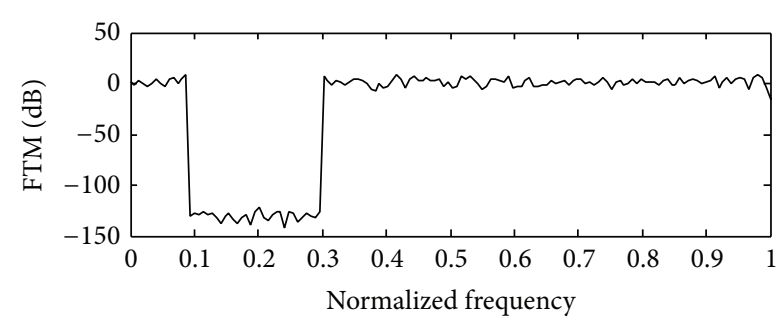

(b)

Figure 5: A design example. (a) The autocorrelation magnitude of the waveform. (b) The spectrum of the waveform.

Here we apply the orthogonal decomposition on $\mathbf{h}$ and $\mathbf{y}$, and the distance between these two vectors can be rewritten as

$$
\operatorname{dist}^{2}(\mathbf{h}, \mathbf{y})=\left\|\mathbf{h}_{\mathbf{s}}-\mathbf{y}_{\mathbf{s}}\right\|^{2}+\left\|\mathbf{h}_{\mathbf{s}}^{\perp}-\mathbf{y}_{\mathbf{s}}^{\perp}\right\|^{2}
$$

where $\mathbf{h}_{\mathbf{s}}=\operatorname{proj}(\mathbf{h}, \operatorname{col}(\mathbf{s})), \mathbf{h}_{\mathbf{s}}^{\perp}=\operatorname{proj}\left(\mathbf{h}, \operatorname{null}\left(\mathbf{s}^{H}\right)\right), \mathbf{y}_{\mathbf{s}}=$ $\operatorname{proj}(\mathbf{y}, \operatorname{col}(\mathbf{s}))$, and $\mathbf{y}_{\mathbf{s}}^{\perp}=\operatorname{proj}\left(\mathbf{y}, \operatorname{null}\left(\mathbf{s}^{H}\right)\right)$. By minimizing the distance, we obtain the projector onto $S_{\text {IPC }}$, shown as follows:

$$
\operatorname{proj}\left(\mathbf{h}, S_{\mathrm{IPC}}(\mathbf{s}, \xi)\right):= \begin{cases}\mathbf{s}, \mathbf{h} & \mathbf{h}=\mathbf{0} \\ \frac{\mathbf{h}}{\|\mathbf{h}\|} & \frac{\mathbf{h}}{\|\mathbf{h}\|} \in S_{\mathrm{IPC}}(\mathbf{s}, \xi) \\ \frac{\xi \mathbf{h}_{\mathbf{s}}}{\left\|\mathbf{h}_{\mathbf{s}}\right\|}+\frac{\sqrt{1-\xi^{2}} \mathbf{h}_{\mathbf{s}}^{\perp}}{\left\|\mathbf{h}_{\mathbf{s}}^{\perp}\right\|}, & \text { otherwise. }\end{cases}
$$

By substituting the projectors into the ISAA framework, the complete form of the algorithm is obtained as follows:

$$
\mathbf{h}_{\text {opt }}=\left(\operatorname{proj}\left(\cdot, S_{\text {IPC }}(\mathbf{s}, \xi)\right) \circ \operatorname{proj}\left(\cdot, S_{\mathrm{CO}}\left(Q_{p}\right)\right)\right)^{K}\left(\mathbf{h}_{\text {init }}\right) .
$$

We name the algorithm ISAA-Cross-correlation Optimization and Inner Product Constraint (COIPC).

\section{Numerical Examples}

4.1. Waveform Design Example. A design example is shown in Figure 5.

The suppressed band is $[0.1,0.3]$ in normalized frequency. From Figure 5 we can find that the power of the waveform in the suppressed band is nearly zero and the autocorrelation is also optimized for mitigating sidelobe interference. The Fourier transform magnitude (FTM) of the waveform is also the frequency response of the matched filter. Consequently, the matched filter can mitigate the interference in the suppressed band without loss of echo power.

4.2. Sea Spikes Suppression. To quantify the clutter suppression performance of the waveform, we define the performance index as follows:

$$
\mathrm{PI}:=\frac{\mathrm{RSI}_{\mathrm{DW}}}{\mathrm{RSI}_{\mathrm{LFM}}}
$$

where $\mathrm{RSI}_{\mathrm{DW}}$ is the range sidelobe interference in the CUT using designed waveform and $\mathrm{RSI}_{\mathrm{LFM}}$ is the range sidelobe

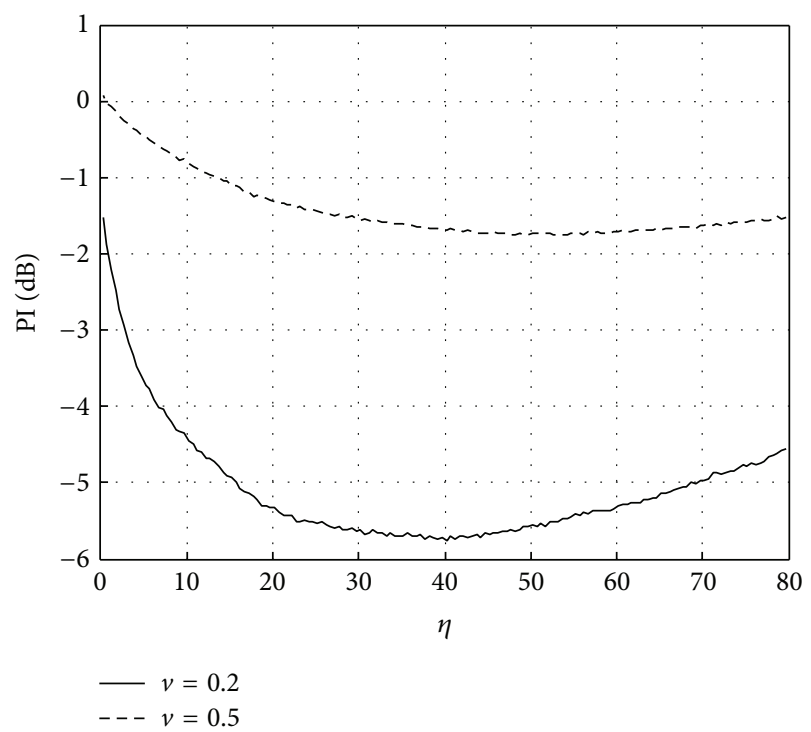

FIgURE 6: Average PI of the designed waveform.

interference in the CUT using LFM. With normalized noise power, the CNR is set to $25 \mathrm{~dB}$, which represents a clutter dominant scene. Figure 6 provides the curve of PI with respect to the CSSA threshold $\eta$. This simulation demonstrates once again that the proposed method is suitable for spiky clutter.

The numerical simulation has shown that this method based on waveform design can mitigate the clutter spikes sidelobe interferences. However, these results are obtained under the assumption that the positions of the spikes are fixed during the processing interval. In fact, sea clutter is highly dynamic; the spikes can only survive for seconds or even shorter, and sometimes they move in range at the group velocity of the sea waves. Thus, compared with mitigating the sidelobes from targets with large RCS, it seems very difficult to apply the method for sea spikes suppression. Here we discuss the scheme and strategy on spikes suppression using specifically designed waveforms.

As mentioned in Section 3.2, the position of the spikes must be determined in order to design the waveform, and this is accomplished by applying the CSSA to the environmental database $\widetilde{Q}_{r}$. Considering that the clutter is dynamic, the database should be updated constantly; otherwise, it will 
provide imprecise information, and the efficiency of the designed waveform will degrade. The radar has to build and update the database itself if other data sources are unavailable, and the authors of [15-17] have provided schemes in this situation. The basic framework of their schemes is dividing each of the dwell durations into several subdwells. In the first subdwell, the radar utilizes a traditional probe waveform and estimates the clutter parameters using the echo returns. After the estimation procedure is finished, the embedded computer of the radar runs the waveform design procedure to synthesize a waveform with spike suppression capability. In the second subdwell, the designed waveform is used for transmitting, and the detection result in this subdwell is considered much reliable compared with the result in the first subdwell because the sidelobe interferences in the CUT are mitigated. Our methods, including CSSA and PONLP, can also be fitted into this framework using CSSA in the first subdwell to determine the position of the spikes, and using the PONLP to design waveform for the second subdwell.

Unfortunately, the scheme discussed above will have no clutter suppression effect if the subdwells cannot be accomplished during the lifetime of the spikes. It may perform even worse than traditional radar for the sidelobes outside the suppressed intervals are sometimes higher than traditional waveforms. In other words, the related algorithms must be effective enough to reduce time consumption. It is obvious that the waveform design algorithm is time-consuming, and thus it is a major concern to improve its computational efficiency. Compared with air-borne and space-borne systems, the limitations of the size and power consumption of the signal processing systems mounted on ship-borne or coastal radars are not so rigorous, which allows us to introduce powerful computers for waveform design. For example, we can build a hybrid system with CPUs and GPUs; these kinds of systems can handle floating point data stream in massive parallel computing. With the floating point operation capability, the computer can reduce the quantization error of the gradients and will converge faster than a fixed point computer. With the parallel computing capability, the time consumption of the gradients calculation can be reduced.

4.3. A Detection Example. We provide a detection example to demonstrate the usage of the methods provided in this paper. We setup a simplified radar scene described as follows:

(i) the signal and interference are normalized with respect to the noise level;

(ii) a large still target with $\sigma^{2}=40 \mathrm{~dB}$ exists in the 100th range cell;

(iii) according to intelligence, a small target may exist near this large target, and its position is unknown to us;

(iv) the echoes of the targets are modeled as Gaussian processes.

Assume that the actual position of the small target is the 84th range cell, and the autocorrelation level of the LFM waveform in this range cell is $-34 \mathrm{~dB}$; thus, the sidelobe interference is $40 \mathrm{~dB}-34 \mathrm{~dB}=6 \mathrm{~dB}$. Firstly, we use the

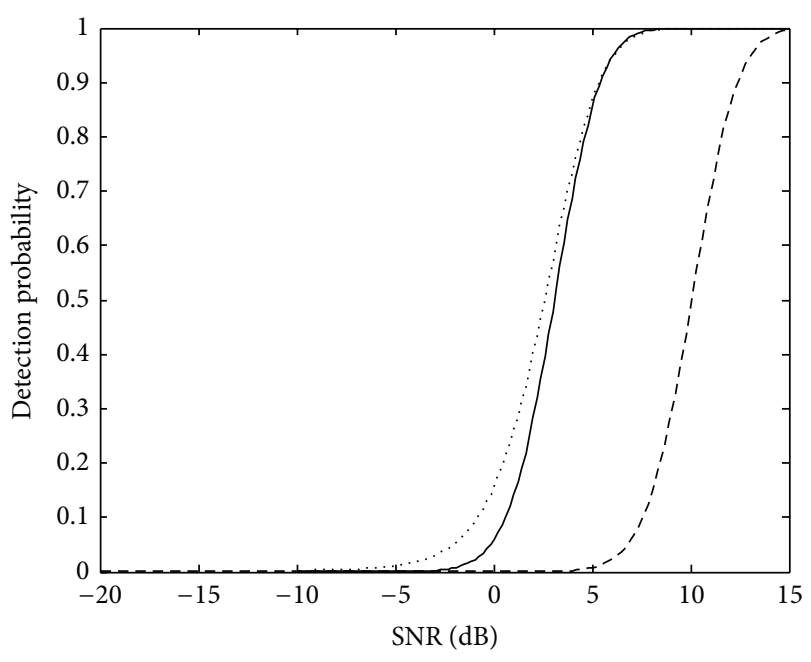

… Normal waveform, mismatched filter, $P_{\mathrm{FA}}=10^{-3}$
- Designed waveform, matched filter, $P_{\mathrm{FA}}=10^{-6}$
- - Normal waveform, matched filter, $P_{\mathrm{FA}}=10^{-6}$

FIGURE 7: The detection performance.

traditional method; that is, pipe the output of the matched filter of 25 consecutive echoes to an energy detector. The false alarm probability is $P_{\mathrm{FA}}=10^{-6}$. The detection probability $P_{\mathrm{D}}$ with respect to $P_{\mathrm{FA}}$ is shown in Figure 7 using dash line.

Secondly, we utilize a two stage method. In the first stage, considering that the position of the small target is unknown, we cannot utilize the method provided in Section 3.1 because the parameter of the set-valued function $Q_{r}$ is not determined. Consequently, we should use the method provided in Section 3.3 to design several receive filters with different correlation nulls to test the intervals adjacent to the large targets. The design examples are shown in Figure 8. The normalized SNR loss is set to $20 \%$.

For the filter shown in Figure 8(b), the correlation of the nulls is lower than $-300 \mathrm{~dB}$, which means that RSI in the 84th range cell is nearly zero. However, the mismatch of the receive filter also introduced $-1.93 \mathrm{~dB}$ signal power lose. In this stage, we set $P_{\mathrm{FA}}=10^{-3}$ which is pretty high to increase the $P_{\mathrm{D}}$ [34]. The detection performance is shown in Figure 7 using dot line.

After the suspicious range of the small target is determined, we start the second stage and run the waveform design procedure. Considering that the small target may have moved, we broaden the suppressed interval. The autocorrelation of the designed waveform is shown in Figure 9. In this stage we use the matched filter of the designed waveform; thus, compared with the previous stage, the mismatched signal power loss is eliminated. To yield a reliable detection result, we set $P_{\mathrm{FA}}=10^{-6}$ in this stage. The detection performance is shown in Figure 7 using solid line. From Figure 7 we can find that in stage II, the detection probability is slightly decreased; however, $P_{\mathrm{FA}}$ is $10^{-6}$, which means that the result is much reliable. Compared with the traditional method (dash line in Figure 7), the detection performance is increased by $7 \mathrm{~dB}$ with the same false alarm probability. 


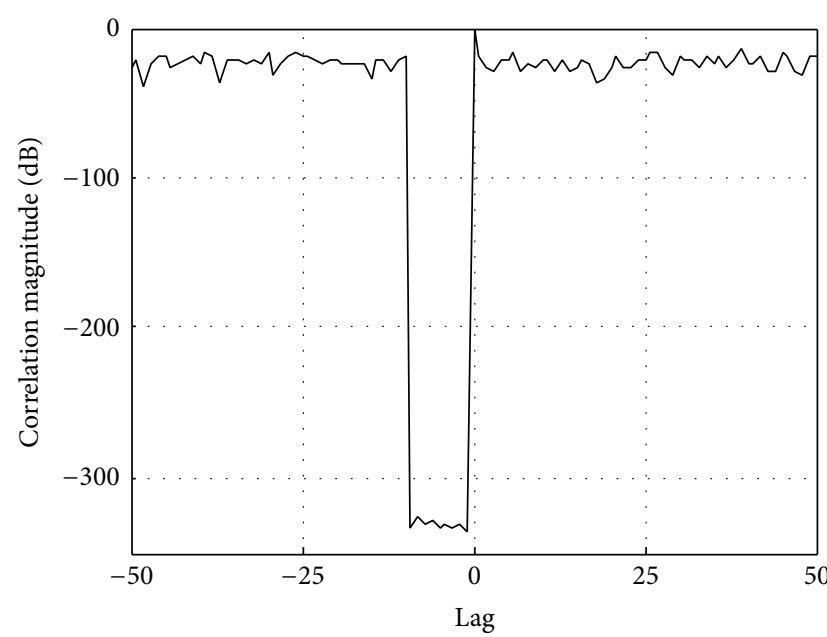

(a)

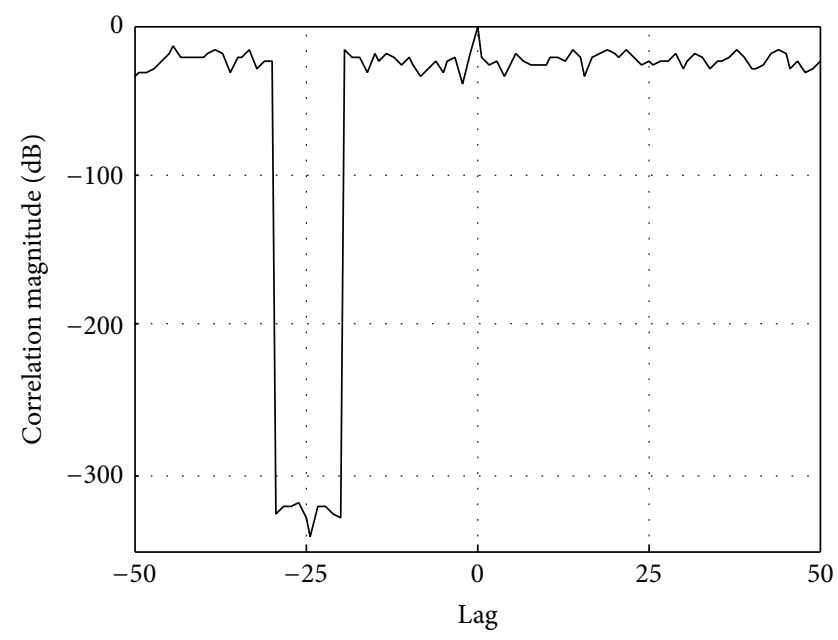

(c)

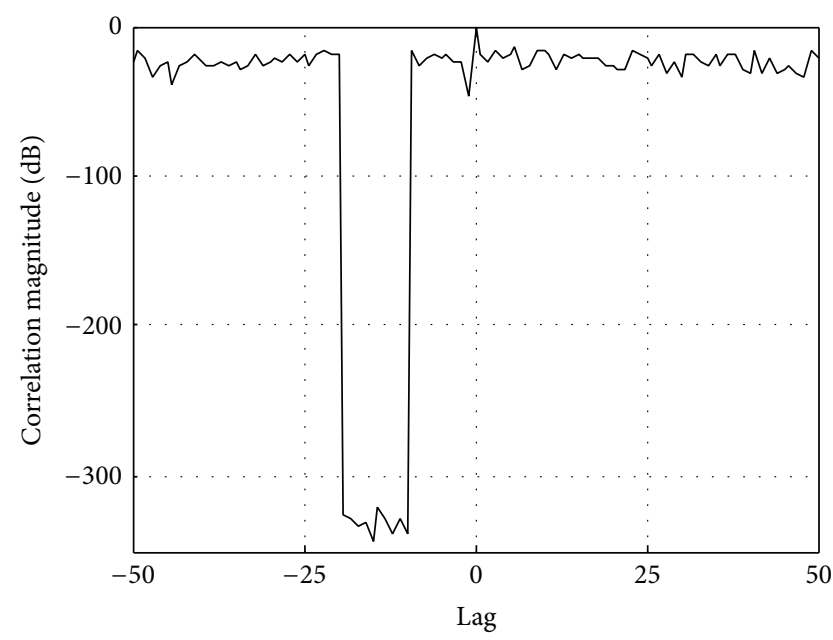

(b)

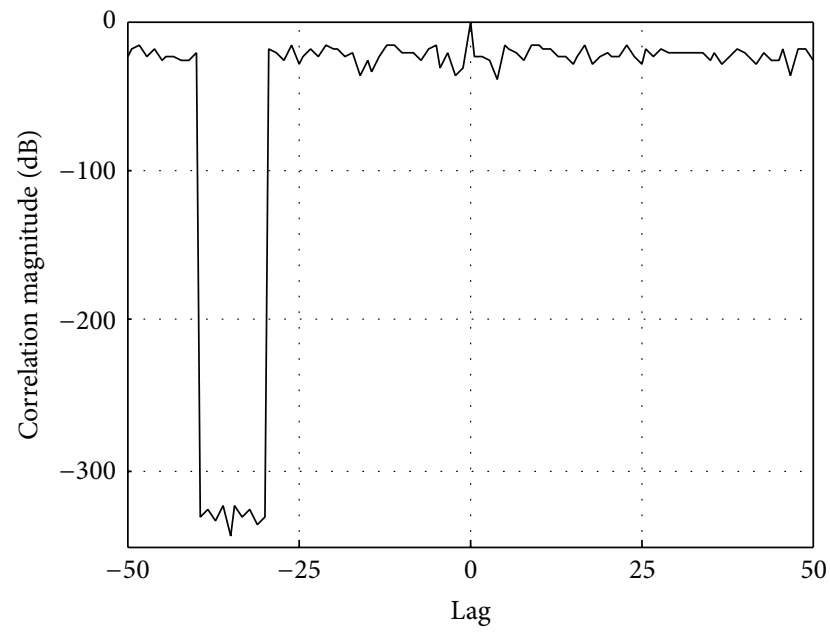

(d)

FiguRE 8: The correlation of the probe waveform and the filter. The suppressed intervals are (a) $[-10,-1],(\mathrm{b})[-20,-11],(\mathrm{c})[-30,-21]$, and (d) $[-40,-31]$.

Notably, the waveform designed here is phase modulated, which means it is Doppler sensitive. This property of the waveform may lead to SNR loss when the radar encounters moving targets. By constructing a receive filter of moving target [35], this problem can be solved. Moreover, our method can make nulls located at specified range-Doppler intervals while the large and the small targets have different velocities, which is demonstrated by the following simulation.

Assume that the small target is staying still as before, while the large target starts to move with its normalized Doppler frequency that equals -0.016 . In the first stage of detection, we design different filters for testing different range Doppler intervals using ISAA-COIPC. One of the filter's discrete cross-ambiguity functions is shown in Figure 10. The suppressed sidelobe zone is located in lag $=[-5,-25]$ and normalized Doppler shift $=-0.016$. After the suspicious range Doppler interval is determined, a waveform is designed using PONLP for the second stage of detection, and its discrete ambiguity function is shown in Figure 11.
From Figures 8, 9, 10, and 11, we can find that ISAACOIPC and PONLP can suppress the sidelobes from a large target located in arbitrary range Doppler cells. However, the filters and waveforms designed in this example may not be realizable in practical systems because the null depth is limited by numerous issues of radar hardware implementation. In fact, the results shown in this example can be considered as the performance upper bound of the proposed method. To evaluate the performance loss caused by practical factors, we apply phase quantization to the designed filters and waveforms. Phase quantization can not only simplify the design and implementation of the radar hardware, but it can also withstand phase noise to some extent. The average null depths after quantization are shown in Table 1.

From Table 1 we can find that the designed filters and waveforms will suffer performance loss after quantization. However, by utilizing these quantized ones, the final detection probability curve nearly coincided with the solid one shown 


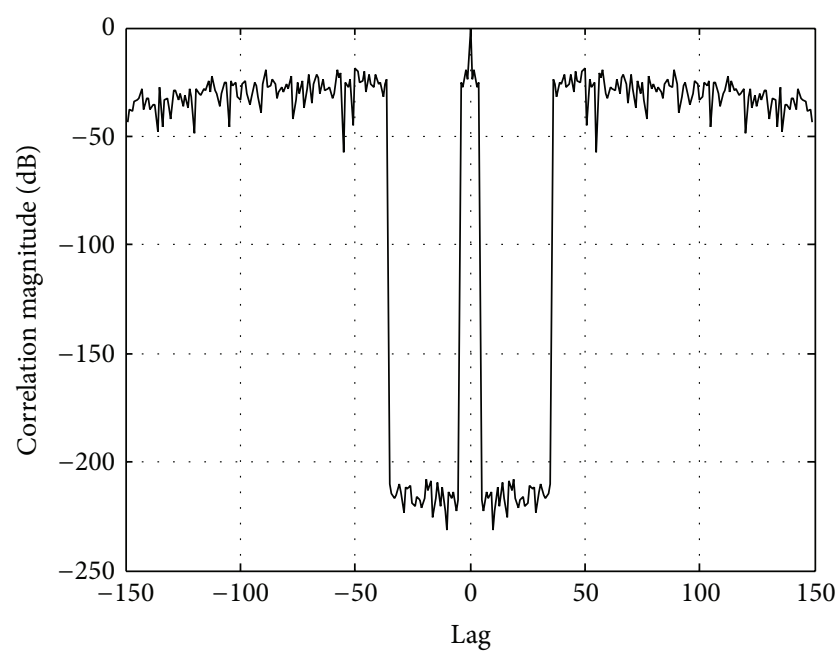

FIGURE 9: The autocorrelation of the designed waveform.

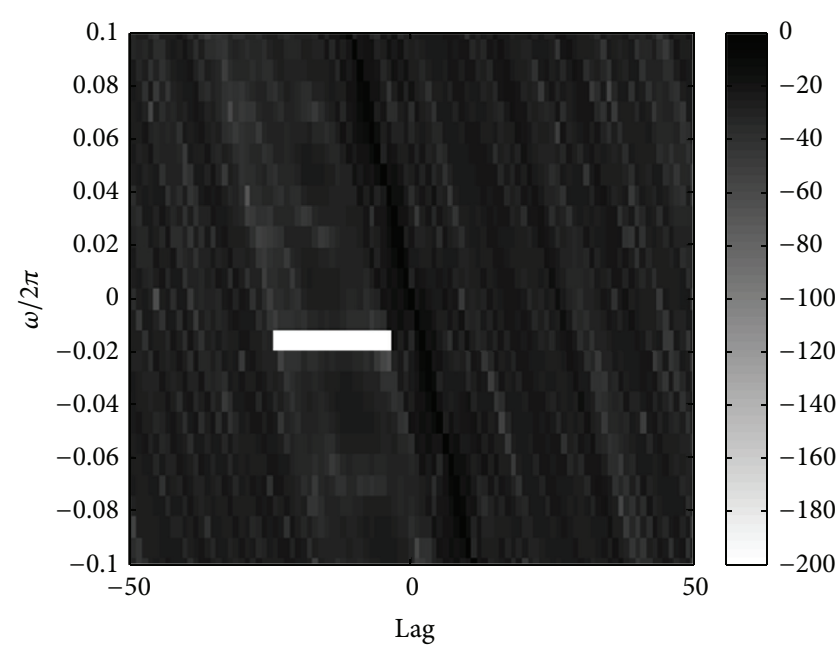

FIGURE 10: The discrete cross-ambiguity function of a designed receive filter.

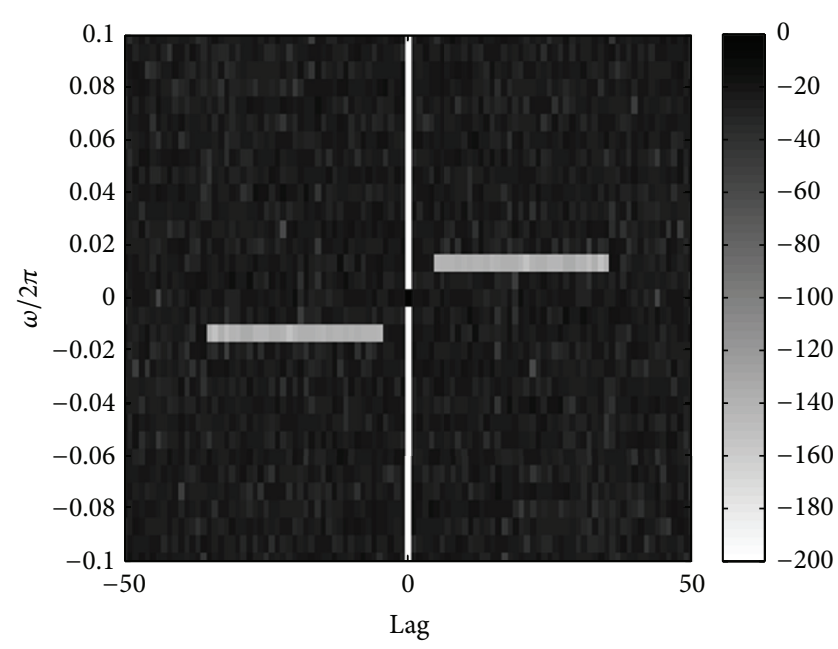

FIGURE 11: The discrete ambiguity function of the designed waveform.
TABLE 1: The average null depths of the quantized filters and waveforms.

\begin{tabular}{lccc}
\hline Quantization (bits) & 8 & 12 & 16 \\
Average null depth of designed filter (dB) & -45 & -61 & -93 \\
Average null depth of designed waveform (dB) & -59 & -88 & -112 \\
\hline
\end{tabular}

in Figure 7. The reason for this phenomenon is that the quantized filters and waveforms are still capable of suppressing sidelobes of the large target efficiently despite the adverse impact of quantization in this example, and the sidelobe interference from the large target becomes trivial for the detector.

\section{Conclusion}

In this paper, we provide a method for designing waveforms and filters with the capability to suppress range sidelobe interference in the CUT from nearby large scatterers and active interference from other wireless devices. By utilizing information from secondary database, the method can suppress the correlation sidelobes in specified zones and can have a stop band in the desired frequency intervals. In scenarios that the secondary database is not adequate to use, the proposed filter design method can be used to determine the zone in which the sidelobe should be suppressed. The numerical examples have shown the usage of these methods, and the result shows that the detection performance is improved about $7 \mathrm{~dB}$ compared with the traditional method under the specified radar scene.

\section{Conflict of Interests}

The authors declare that there is no conflict of interests regarding the publication of this paper.

\section{Acknowledgments}

This work is supported by the National Natural Science Foundation of China (61371181), Shandong Provincial Natural Science Foundation, China (ZR2012FQ007), and the Natural Scientific Research Innovation Foundation in Harbin Institute of Technology (HIT.NSRIF.2011118).

\section{References}

[1] R. V. de Jongh, "Naval radar in a littoral environment," in Proceedings of the IEEE MTT-S International Microwave Symposium, pp. 1457-1460, June 2005.

[2] D. G. Money, J. Branson, M. Hooker, and A. Mabogunje, "Radar littoral environment predictions and measurements," in Proceedings of the Radar Edinburgh International Conference (Radar '97), pp. 164-166, October 1997.

[3] F. R. Castella and J. R. Moore, "Improved target detection in a littoral environment," in Proceedings of the Radar Edinburgh International Conference (Radar '97), pp. 429-433, October 1997. 
[4] K. D. Ward and R. J. A. Tough, "Modelling radar sea clutter in littoral environments," in Proceedings of the International Conference on Radar (Radar '08), pp. 82-87, September 2008.

[5] J. E. Cilliers and J. C. Smit, "Pulse compression sidelobe reduction by minimization of $\mathrm{L}_{\mathrm{p}}$-norms," IEEE Transactions on Aerospace and Electronic Systems, vol. 43, no. 3, pp. 1238-1247, 2007.

[6] S. D. Blunt and K. Gerlach, "Adaptive pulse compression via MMSE estimation," IEEE Transactions on Aerospace and Electronic Systems, vol. 42, no. 2, pp. 572-584, 2006.

[7] S. M. Sussman, "Least-square synthesis of radar ambiguity functions," IRE Transactions on Information Theory, vol. 8, no. 3, pp. 246-254, 1962.

[8] M. Ruggiano, E. Stolp, and P. van Genderen, "Multi-target performance of LMMSE filtering in radar," IEEE Transactions on Aerospace and Electronic Systems, vol. 48, no. 1, pp. 170-179, 2012.

[9] E. de Witte and H. D. Griffiths, "Improved ultra-low range sidelobe pulse compression waveform design," Electronics Letters, vol. 40, no. 22, pp. 1448-1450, 2004.

[10] F. F. Kretschmer Jr. and K. Gerlach, "Low sidelobe radar waveforms derived from orthogonal matrices," IEEE Transactions on Aerospace and Electronic Systems, vol. 27, no. 1, pp. 92-102, 1991.

[11] M. Soltanalian, M. M. Naghsh, and P. Stoica, "A fast algorithm for designing complementary sets of sequences," Signal Processing, vol. 93, no. 7, pp. 2096-2102, 2013.

[12] Y.-C. Wang, L. Dong, X. Xue, and K.-C. Yi, "On the design of constant modulus sequences with low correlation sidelobes levels," IEEE Communications Letters, vol. 16, no. 4, pp. 462-465, 2012.

[13] P. Stoica, H. He, and J. Li, "New algorithms for designing unimodular sequences with good correlation properties," IEEE Transactions on Signal Processing, vol. 57, no. 4, pp. 1415-1425, 2009.

[14] M. Soltanalian and P. Stoica, "Computational design of sequences with good correlation properties," IEEE Transactions on Signal Processing, vol. 60, no. 5, pp. 2180-2193, 2012.

[15] T. Zhang, G. Cui, L. Kong, W. Yi, and X. Yang, "Phasemodulated waveform evaluation and selection strategy in compound-Gaussian clutter," IEEE Transactions on Signal Processing, vol. 61, no. 5, pp. 1143-1148, 2013.

[16] H. T. Wang, L. Shi, Y. L. Wang, and D. Ben, "A novel target detection approach based on adaptive radar waveform design," Chinese Journal of Aeronautics, vol. 26, no. 1, pp. 194-200, 2013.

[17] S. P. Sira, D. Cochran, A. Papandreou-Suppappola et al., "Adaptive waveform design for improved detection of low-RCS targets in heavy sea clutter," IEEE Journal on Selected Topics in Signal Processing, vol. 1, no. 1, pp. 56-66, 2007.

[18] A. Benavoli, L. Chisci, A. Farina, S. Immediata, L. Timmoneri, and G. Zappa, "Knowledge-based system for multi-target tracking in a littoral environment," IEEE Transactions on Aerospace and Electronic Systems, vol. 42, no. 3, pp. 1100-1116, 2006.

[19] A. Aubry, A. D. Maio, A. Farina, and M. Wicks, "Knowledgeaided (potentially cognitive) transmit signal and receive filter design in signal-dependent clutter," IEEE Transactions on Aerospace and Electronic Systems, vol. 49, no. 1, pp. 93-117, 2013.

[20] F. Li, Y. Zhao, and X. Qiao, "A waveform design method for suppressing range sidelobes in desired intervals," Signal Processing, vol. 96, pp. 203-211, 2014.

[21] L. K. Patton and B. D. Rigling, "Phase retrieval for radar waveform optimization," IEEE Transactions on Aerospace and Electronic Systems, vol. 48, no. 4, pp. 3287-3302, 2012.
[22] J. Hu and J. Gao, "Multiscale characterization of sea clutter by scale-dependent Lyapunov exponent," Mathematical Problems in Engineering, vol. 2013, Article ID 584252, 9 pages, 2013.

[23] G. L. Turin, "An introduction to matched filters," IRE Transactions on Information Theory, vol. 6, no. 3, pp. 311-329, 1960.

[24] S. Kay, "Optimal signal design for detection of Gaussian point targets in stationary Gaussian clutter/reverberation," IEEE Journal on Selected Topics in Signal Processing, vol. 1, no. 1, pp. 31-41, 2007.

[25] G. Wang and Y. Lu, "Designing single/multiple sparse frequency waveforms with sidelobe constraint," IET Radar, Sonar and Navigation, vol. 5, no. 1, pp. 32-38, 2011.

[26] M. J. Lindenfeld, "Sparse frequency transmit and receive waveform design," IEEE Transactions on Aerospace and Electronic Systems, vol. 40, no. 3, pp. 851-861, 2004.

[27] S. Watts, "Modeling and simulation of coherent sea clutter," IEEE Transactions on Aerospace and Electronic Systems, vol. 48, no. 4, pp. 3303-3317, 2012.

[28] K. J. Sangston, F. Gini, and M. S. Greco, "Coherent radar target detection in heavy-tailed compound-gaussian clutter," IEEE Transactions on Aerospace and Electronic Systems, vol. 48, no. 1, pp. 64-77, 2012.

[29] S. Watts, "Radar detection prediction in sea clutter using the compound K-distribution model," IEE Proceedings F, vol. 132, no. 7, pp. 613-620, 1985.

[30] S. Haykin, R. Barker, and B. W. Currie, "Uncovering nonlinear dynamics-the case study of sea clutter," Proceedings of the IEEE, vol. 90, no. 5, pp. 860-881, 2002.

[31] S. T. Smith, “Optimum phase-only adaptive nulling," IEEE Transactions on Signal Processing, vol. 47, no. 7, pp. 1835-1843, 1999.

[32] F. Posner and K. Gerlach, "Sea spike demographics at high range resolutions and very low grazing angles," in Proceedings of the IEEE Radar Conference, pp. 38-45, May 2003.

[33] M. Greco, P. Stinco, and F. Gini, "Identification and analysis of sea radar clutter spikes," IET Radar, Sonar and Navigation, vol. 4, no. 2, pp. 239-250, 2010.

[34] A. Aubry, A. de Maio, B. Jiang, and S. Zhang, "Ambiguity function shaping for cognitive radar via complex quartic optimization," IEEE Transactions on Signal Processing, vol. 61, no. 22, pp. 5603-5619, 2013.

[35] M. A. Richards, Fundamentals of Radar Signal Processing, McGraw-Hill, New York, NY, USA, 2005. 


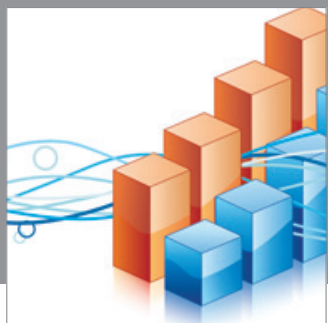

Advances in

Operations Research

mansans

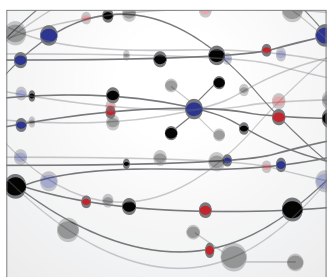

The Scientific World Journal
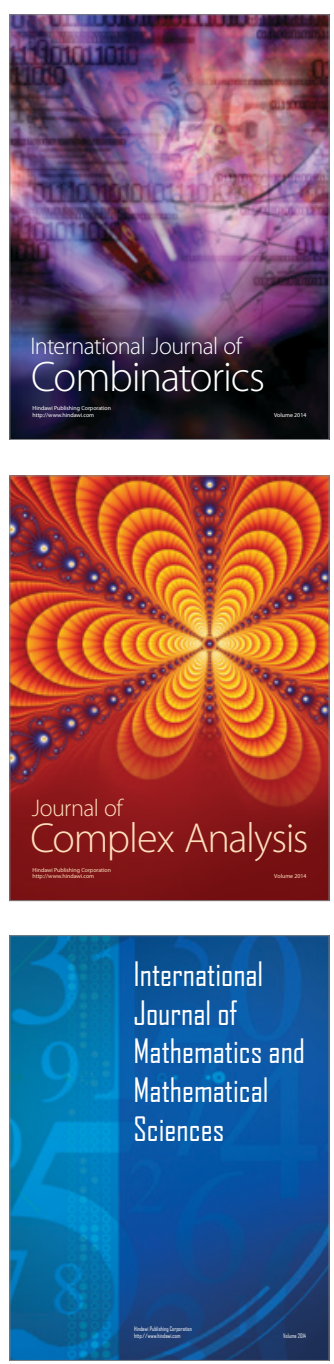
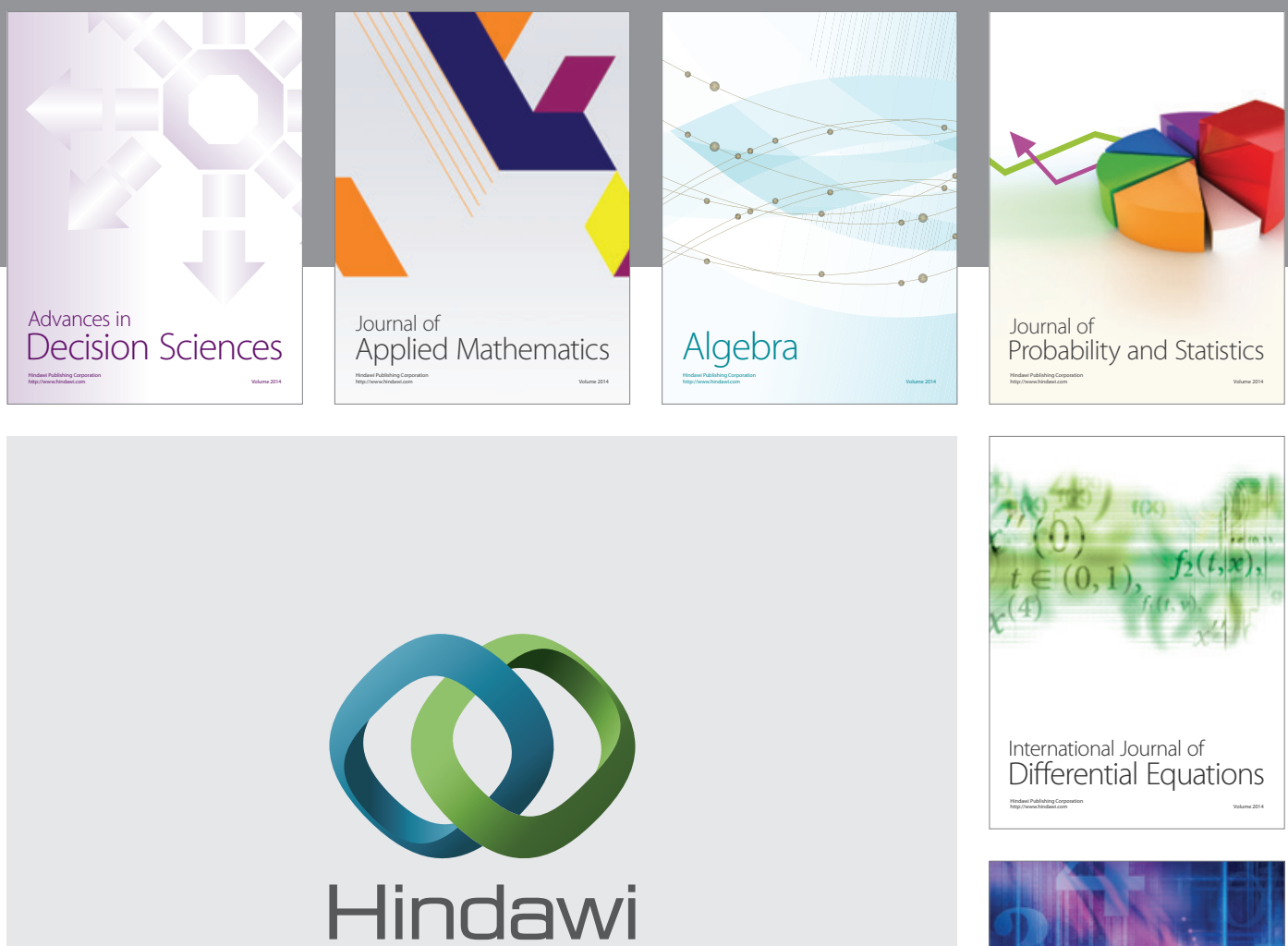

Submit your manuscripts at http://www.hindawi.com
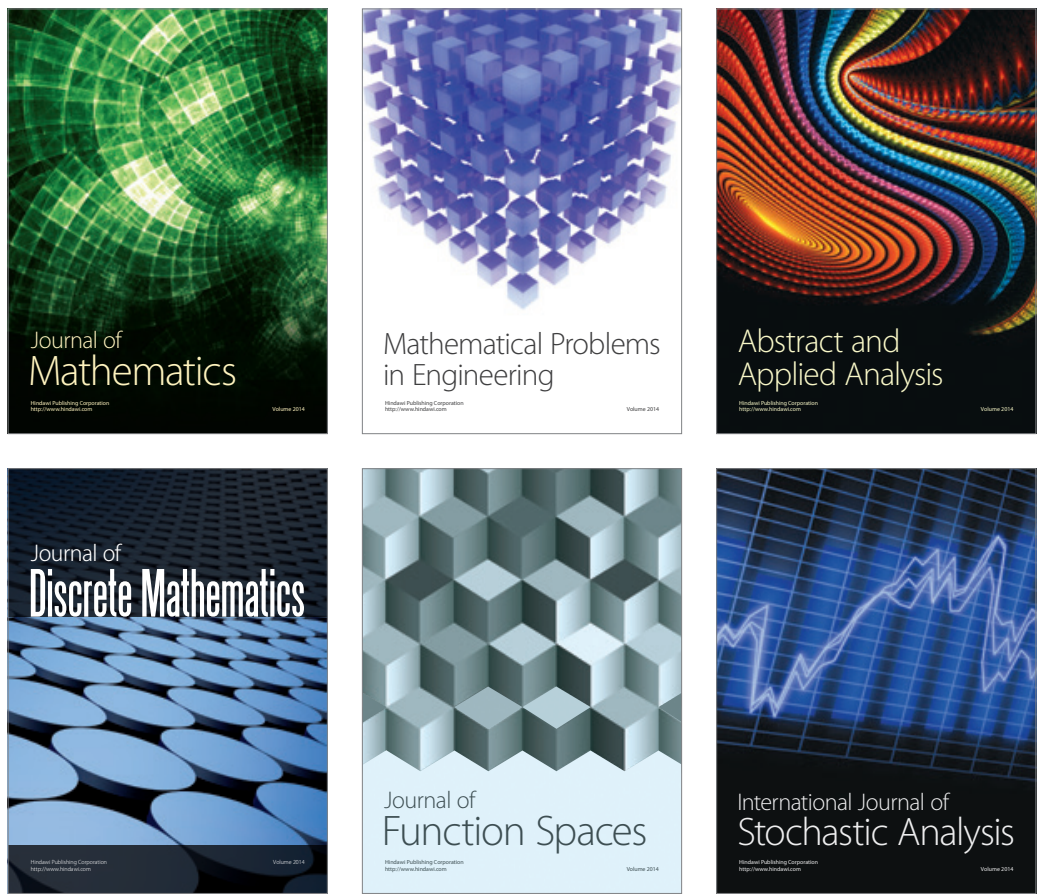

Journal of

Function Spaces

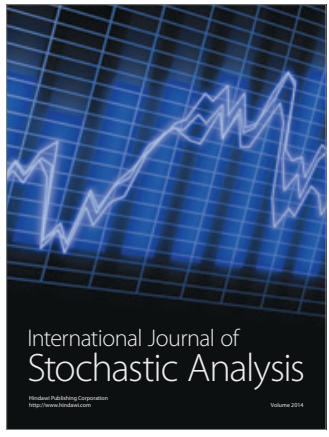

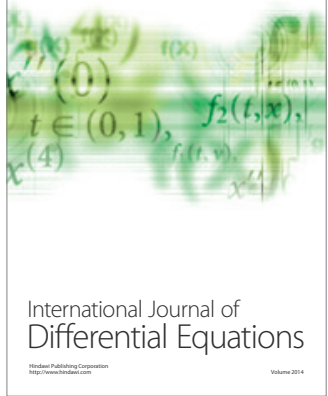
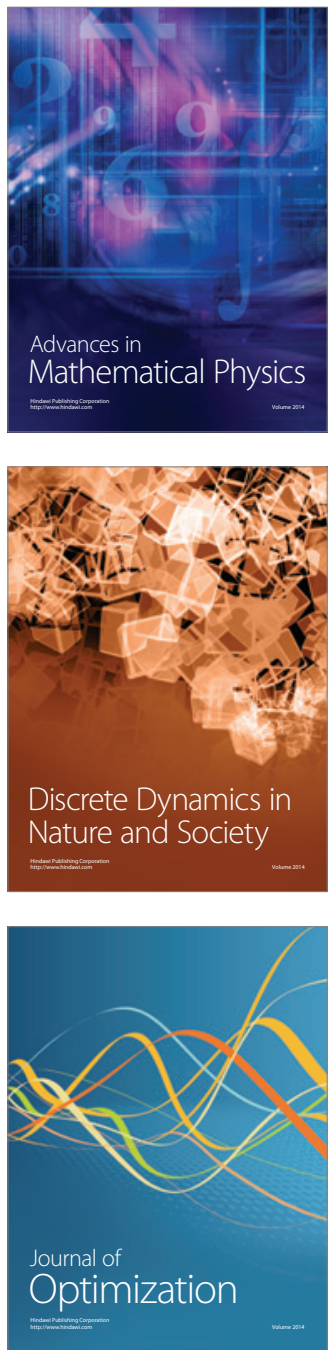